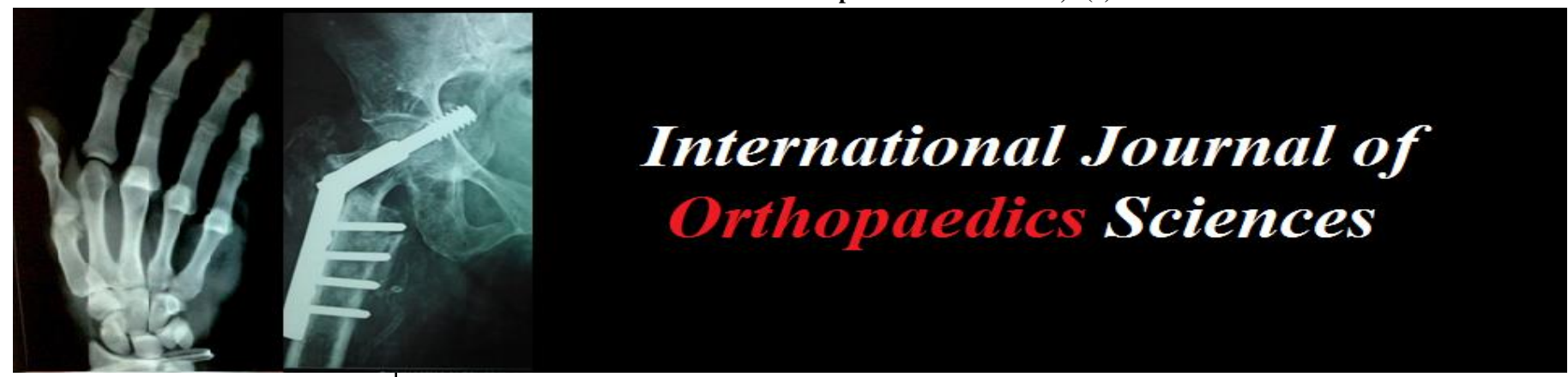

ISSN: $2395-1958$

IJOS 2018; 4(3): 314-321

(C) 2018 IJOS

www.orthopaper.com

Received: 27-05-2018

Accepted: 28-06-2018

Dr. Prashant Pandey

Resident Orthopaedics, Krishna

Medical College, karad,

Maharashtra, India

Dr. Sandip Patil

Associate Professor, Krishna

Medical College, Karad,

Maharashtra, India

Dr. Nishant Gaonkar

Assistant Professor, Krishna

Medical College, Karad,

Maharashtra, India

Dr. Vijay Sinh Patil

Senior Resident Orthopaedics,

Krishna Medical College, Karad,

Maharashtra, India

Dr. Rutvik A Shah

Resident Orthopaedics, Krishna

Medical College, Karad,

Maharashtra, India

Correspondence

Dr. Prashant Pandey

Resident Orthopaedics, Krishna

Medical College, Karad,

Maharashtra, India

\section{A comparative study of functional outcomes of fractures treated with plating versus conservative for complex proximal humerus fractures in elderly}

\author{
Dr. Prashant Pandey, Dr. Sandip Patil, Dr. Nishant Gaonkar, Dr. Vijay \\ Sinh Patil and Dr. Rutvik A Shah
}

DOI: https://doi.org/10.22271/ortho.2018.v4.i3f.57

\section{Abstract}

Introduction: Most proximal humerus fractures are minimally displaced, low energy osteoporotic fractures and are effectively treated with conservative treatment. But for those that have moderate to severe displacement, the optimal treatment for the patient has not been fully elucidated.

Aims and Objectives: To compare and evaluate functional outcome of complex proximal humerus fractures treated with plating and conservative method in elderly.

Materials and methods: A prospective, comparative study was conducted with 50 patients to compare and evaluate functional outcome of complex proximal humerus fractures treated with plating and conservative method in elderly.

Results: The mean flexion, abduction, external rotation and internal rotation values of conservative group were significantly lesser as compared to operative group after 6 weeks. However there was no significant difference between the groups after 6 months.

Conclusion: Locking plate is an implant of choice for comminuted proximal humerus fractures.In the present study, the outcomes of locked plate fixation were similar to those of nonoperative treatment. Early physiotherapy and good rehabilitation programme is vital to get a good functional outcome.

Keywords: Proximal humerus, elderly, plate, conservative

\section{Introduction}

Proximal humerus fractures are seen most commonly in the elderly population, following a low energy fall ${ }^{[1,2]}$. For the most part, proximal humerus fractures can be treated conservatively with the anticipation that the fracture will heal and the patient will regain function in that shoulder. Most proximal humerus fractures are minimally displaced, low energy osteoporotic fractures and are effectively treated with conservative treatment. But for those that have moderate to severe displacement, the optimal treatment for the patient has not been fully elucidated. The treatment algorithm has continued to evolve. There have been improvements to the design, technology, and techniques for operative fixation and arthroplasty. Also, patients are now living longer and leading more active lifestyles. Their expectations for outcome have changed, and using chronologic age to categorize these fractures is becoming less relevant. The goals of treatment should be to maximize the function of the shoulder and to minimize the chance of treatment failure, all while working within the expectations of the patient.

Proximal humerus fractures represent approximately 5\% of all fractures and occur in a bimodal frequency with greatest incidence in the elderly population ${ }^{[1,3,4]}$. More than $70 \%$ of patients with these fractures are older than 60 years of age, $75 \%$ are women, and the fractures are often related to osteoporosis ${ }^{[5,6]}$. For this elderly population the goal of treatment of proximal humeral fractures is to maintain independence of daily living by achieving a painless shoulder with an adequate function.

Roughly $80 \%$ of these fractures are minimally displaced and can be successfully treated with non-operative management. In the remaining $20 \%$, fracture fragments are displaced and may exhibit an unstable fracture pattern. Cases where there is marked displacement, and instability 
are challenging for the operating surgeon, non-operative treatment can yield less than satisfactory results and operative fixation is often indicated ${ }^{[7,8]}$. Non-operative treatment results in minimal pain but with reduced function reportedly comparable to that of shoulder fusion ${ }^{[8]}$. A wide variety of fixation devices have been developed over the past several decades to treat displaced and unstable proximal humerus fractures ${ }^{[7,8]}$, In our review of the literature, however, we found there is little agreement on the choices of treatment of these complex fractures. Furthermore, the incidence of complications from surgery reportedly varies between $11 \%$ and $50 \%[9,10]$.

Treatment of proximal humeral fractures has been the subject of much controversy and confusion This is because of the complexity of these injuries, the fracture displacements are difficult to see without careful radiographic views and also due to associated soft tissue injuries. Further, there has always been diversity of opinion about the care of shoulder fractures, with frequent controversies and lively debates, furthermore even good anatomical results achieved at operative repair lead to poor results unless there is meticulous post-operative rehabilitation, which can be more challenging in the shoulder than operative technique ${ }^{[32-33]}$

Hence the present study was done at our tertiary care centre to compare and Evaluate Functional Outcome of Complex Proximal Humerus Fractures treated with Plating and Conservative Method In Elderly.

\section{Material and Methods}

A prospective, comparative study was conducted with 50 patients to compare and evaluate functional outcome of complex proximal humerus fractures treated with plating and conservative method in elderly. The patients were selected randomly and were divided in the following two groups of 25 patients each:

Group A: Cases of complex proximal humerus fractures treated conservatively.

Group B: Cases of complex proximal humerus fractures treated with plating.

Study Design: It will be a prospective and comparative clinical study.

Study Duration: June 2015 to December 2016 at a tertiary health care centre.

\section{Sample Size}

50 patients to compare and evaluate functional outcome of complex proximal humerus fractures treated with plating and conservative method in elderly. The patients were selected randomly and were divided in the following two groups of 25 patients each:

Group A: Cases of complex proximal humerus fractures treated conservatively

Group B: Cases of complex proximal humerus fractures treated with plating.
At alpha 0.05 , we calculated that 20 patients per group would provide $90 \%$ power to detect a $20 \%$ increase in the treatment groups. However to allow for the comparisons between the control group and each treatment group, an adjusted $p$ (Bonferroni correction) of 0.025 was considered significant for the primary outcome and the required sample size increased to 25 per group.

\section{Inclusion criteria}

Patients above 50 years with complex proximal humerus fractures including three-part and four-part fractures.

\section{Both Sex}

Medically fit and Willingness of the Patient to Participate and to undergo surgery.

Patients treated conservatively coming for regular followup.

\section{Exclusion criteria}

- Skeletally immature patients.

- Pathological fractures.

- Patient with distal neurovascular deficit.

- Shaft humerus fracture with proximal extension

- Fracture dislocation of shoulder joint

\section{Methodology}

\section{Diagnosis of patients}

The diagnosis of fractures of complex proximal humerus fracture was done purely on $\mathrm{X}$ - rays.

The subjects in the study, who have fulfilled inclusion and exclusion criteria, were selected for the study.

\section{Materials used}

- Well Equipped Operation Theatre With Image Intensifier

- All Basic Orthopaedic instruments

- Plates

- $\quad$ Locking/Non locking

- Dynaplast

- Shoulder immobiliser

- Shoulder pouch

All the facilities, equipment's required for the present study were available in our institution.

After approval from the ethical committee, written Informed Consent was taken.

On admission, a detailed history including the complaints of the patient and the mechanism of injury were noted. Clinical examination involved assessing pain, swelling, tenderness, crepitus and ecchymosis around the shoulder region. A detailed neurovascular examination of the brachial plexus and axillary artery was carried out. Associated injuries to the chest, abdomen and other limbs were also noted. Radiographs of affected shoulder were taken in antero-posterior and axillary views. For primary treatment, immobilization was given in the form of simple cuff and collar sling. All routine investigations were done prior to anaesthesia fitness. Preoperative anaesthesia fitness was done. Patients to be managed operatively were posted for planned operative procedure. 


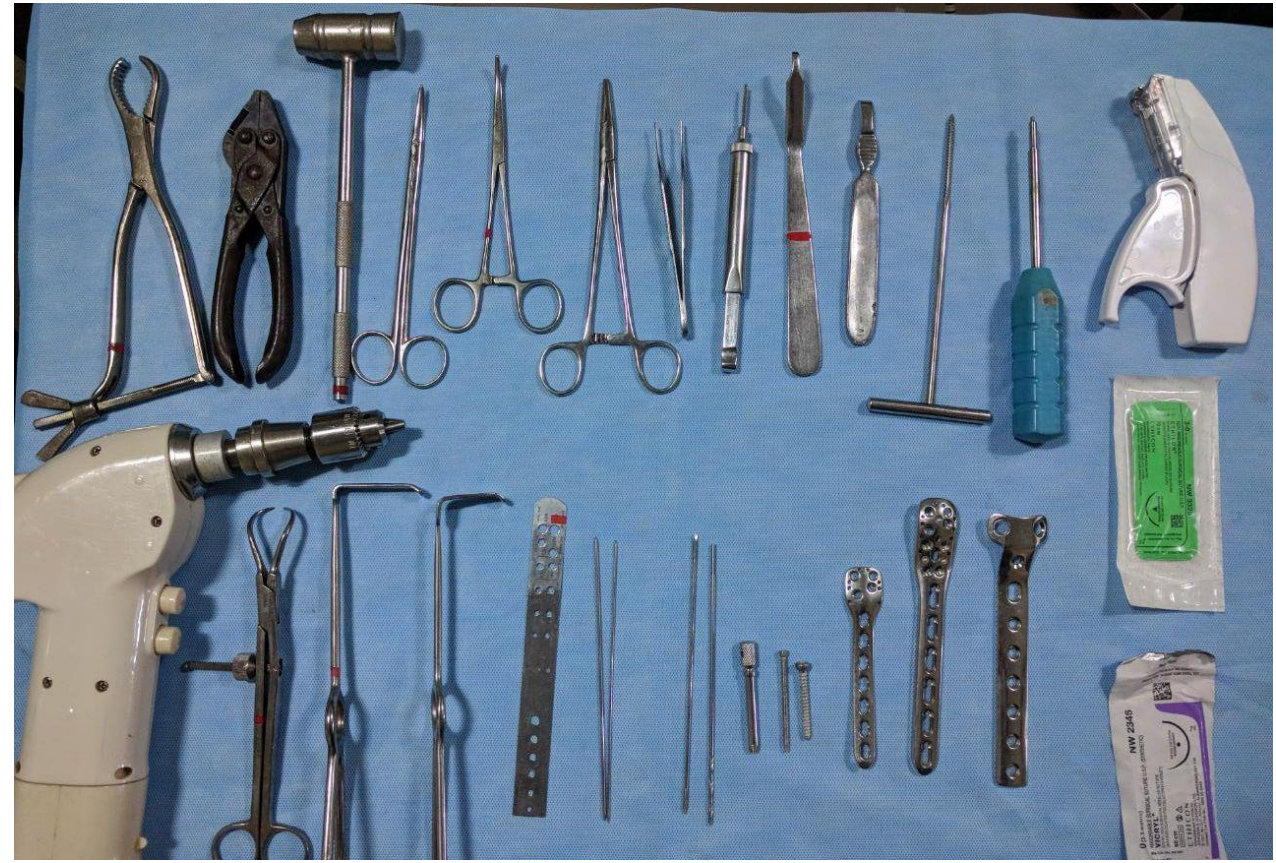

Fig 5: Instruments and implants

\section{Conservative management}

Immediate immobilization was done using a shoulder arm pouch with immobilizer or strapping. Oral analgesics and calcium supplements were given. Physiotherapy in the form of gentle passive range of motion and pendulum exercises was started after 3-4 weeks, once pain was reduced and the patient co-operated. At 6 weeks and 6 months X-ray shoulder antero-posterior and axillary views were taken. On clinical and radiological assessment both active and passive shoulder range of motion exercises were started.

\section{Operative management}

Following pre-operative X-ray shoulder antero-posterior and axillary views and baseline investigations, patients were posted for open reduction and internal fixation with locking compression plate.

\section{Surgical technique}

A beach-chair position was given to the patient, following general anaesthesia. The $\mathrm{C}$-arm was positioned properly to view the proximal humerus. Scrubbing, painting and draping of the affected upper limb were done under aseptic precautions. Deltoid-splitting approach was used to expose the proximal humerus. Care was taken not to injure the axillary nerve. When the fracture was exposed, the humeral head if dislocated, was relocated first into the glenoid under C-arm guidance. Next, the head was reduced onto the shaft with the help of K-wires and then using them as joysticks reduction was achieved in coronal, sagittal and horizontal planes. Remaining fragments were then reduced with the help of traction sutures placed in the rotator cuff insertions. Once an acceptable reduction was achieved, the locking compression plate was applied at least $1 \mathrm{~cm}$ distal to upper end of the greater tubercle and fixed to the humeral shaft with cortical screws. An aiming device which diverged the screws in the head was temporarily attached to the upper part of the plate. Locking screws were then inserted in the humeral head. After obtaining a stable plate fixation, the K-wires and traction sutures were removed. Suturing was done in layers, followed by sterile dressing.

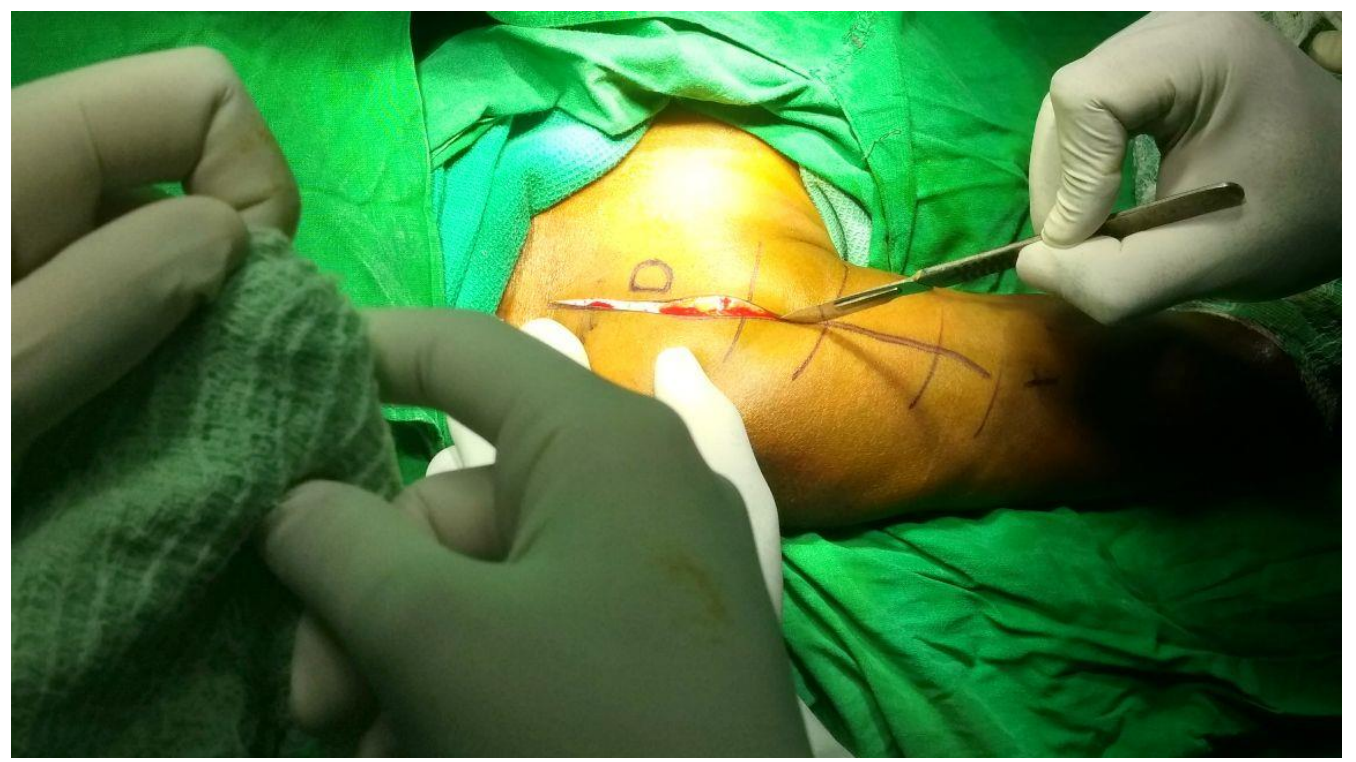

Fig 6: Intra operative Photograph A 


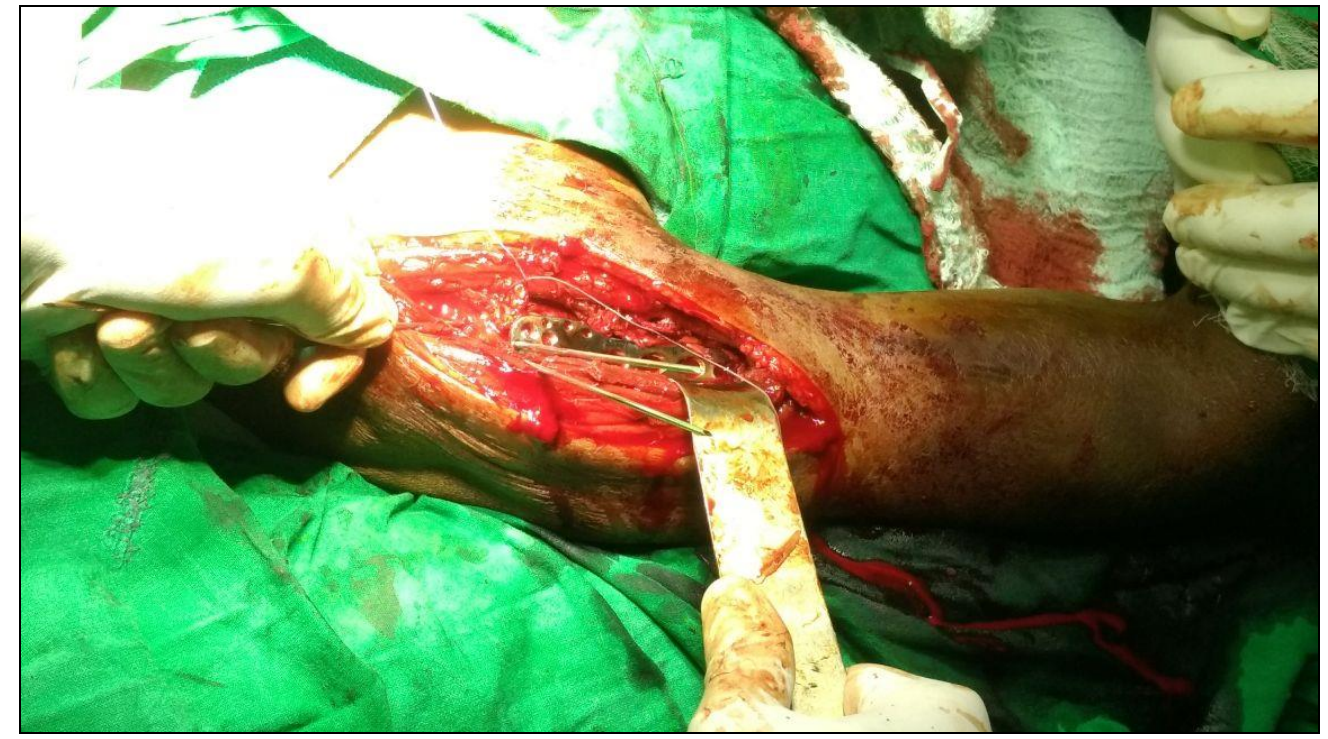

Fig 7: Intra operative Photograph B

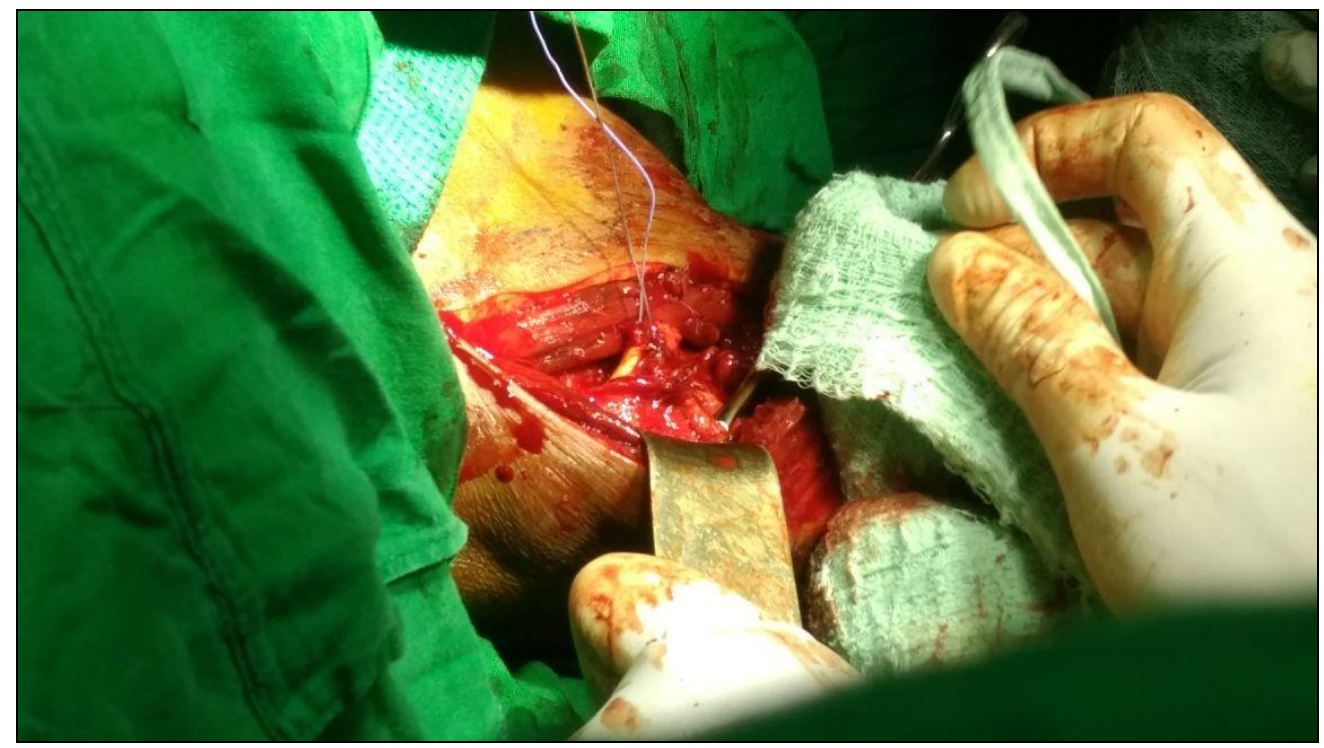

Fig 8: Intra operative Photograph C

\section{Post-operative management}

Limb was immobilised in a shoulder arm pouch. Immediate post-operative X-rays of shoulder in anteroposterior and axillary views were taken to assess reduction of fracture and stability of fixation.

Intravenous antibiotics were given for the first 3 days and then shifted to oral antibiotics. Anti-inflammatory and analgesic drugs were also given. Post-operative dressings of the surgical wound were done on 2 nd and $8^{\text {th }}$ day. Sutures were removed on 12th post-operative day. Mobilisation of affected shoulder was started on the $3^{\text {rd }}$ day with pendulum exercises as per the patient's tolerance. At 6 weeks and 6 months X-ray of the shoulder in antero-posterior and axillary views were taken. On clinical and radiological assessment both active and passive shoulder range of motion exercises were started.

\section{Observation and results}

A prospective, comparative study was conducted with 50 patients to compare and evaluate functional outcome of complex proximal humerus fractures treated with plating and conservative method in elderly. The patients were selected randomly and were divided in the following two groups of 25 patients each:
Group A: Cases of complex proximal humerus fractures treated conservatively

Group B: Cases of complex proximal humerus fractures treated with plating

The following observations were noted:

1. Majority of the patients $(36 \%)$ in Group A were from the age group of $61-70$ years followed by $28 \%$ from the age group of 71-80 years, $20 \%$ from the age group of $81-90$ years and $16 \%$ from the age group of 51-60 years. The mean age in Group A was $69.4 \pm 9.78$ years. Majority of the patients $(40 \%)$ in Group B were from the age group of $61-70$ years followed by $20 \%$ from the age group of 51-60 years and $13.3 \%$ each from the age groups of 7180 years and 81-90 years. The mean age in Group B was $68.7 \pm 10.07$ years. As per Student $t$ test, there was no significant association between the groups $(p>0.05)$.

2. Majority of the patients in both groups were male. There were $80 \%$ and $84 \%$ male patients in Group A and Group B respectively whereas female patients constituted $20 \%$ and $16 \%$ of the study group respectively. There was no significant association between the groups as per ChiSquare test $(p>0.05)$.

3. There was dominance of left side $(60 \%$ and $64 \%)$ as 
compared to right side (40\% and $36 \%$ ) in both groups. There was no significant association between the groups as per Chi-Square test $(p>0.05)$.

4. Road Traffic Accident was observed to be the main cause of fracture in both the groups $(60 \%$ and $64 \%$ respectively) followed by fall (40\% and $36 \%$ respectively). There was no significant association between the groups as per Chi-Square test $(p>0.05)$.

5. According to Neer classification, $52 \%$ and $56 \%$ patients in Group A and Group B respectively belonged to 3 part fracture group while $48 \%$ and $44 \%$ patients in Group A and Group B respectively belonged to 4 part fracture group. There was no significant association between the groups as per Chi-Square test ( $p>0.05)$.

6. $20 \%$ and $12 \%$ patients in Group A had Diabetes Mellitus and Hypertension respectively whereas $16 \%$ and $24 \%$ patients in Group B had Diabetes Mellitus and Hypertension respectively. There was no significant association between the groups as per Chi-Square test $(p>0.05)$.

7. $5(20 \%)$ and $4(16 \%)$ patients in Group A and Group B respectively had associated injuries. There was no significant association between the groups as per ChiSquare test $(p>0.05)$.

8. In Group $\mathrm{A}$, the duration of trauma to treatment in 16 (64\%) and 5 (20\%) patients was 1-3 and 4-6 days respectively and it was 7-9 and 10-11 days in 2 (8\%) patients each respectively. The mean duration of trauma to surgery was $4.04 \pm 2.67$ days. In Group B, the duration of trauma to treatment in $15(60 \%)$ and $4(16 \%)$ patients was 1-3 and 4-6 days respectively and it was 7-9 and 1011 days in $2(8 \%)$ and $4(16 \%)$ patients respectively. The mean duration of trauma to surgery was $4.24 \pm 3.26$ days. As per Student t-test, there was no significant association between the groups $(p>0.05)$.

9. In Group A, the duration to clinical union in $15(60 \%)$ and $6(24 \%)$ patients was 10-13 and 14-17 weeks respectively and it was $18-20$ weeks in $4(16 \%)$ patients. The mean duration for clinical union in Group A was 13.2 weeks. In Group B, the duration to clinical union in $16(64 \%)$ and $5(20 \%)$ patients was $10-13$ and $14-17$ weeks respectively and it was 18-20 weeks in $4(16 \%)$ patients. The mean duration for clinical union in Group B was 13.4 weeks. There was no significant association between the groups as per Chi-Square test $(p>0.05)$.

10. In Group $\mathrm{A}$, the duration to radiological union in 16 $(64 \%)$ and $7(28 \%)$ patients was $17-19$ and 20-22 weeks respectively and it was $23-25$ weeks in $2(8 \%)$ patients. The mean duration for radiological union in Group A was 19.3 weeks. In Group B, the duration to radiological union in $17(68 \%)$ and $5(20 \%)$ patients was 17-19 and 20-22 weeks respectively and it was 23-25 weeks in 3 (12\%) patients. The mean duration for radiological union in Group B was 19.1 weeks. There was no significant association between the groups as per Chi-Square test $(p>0.05)$.

11. During the 6 weeks follow up period, $3(12 \%)$ patients of Group A and $2(8 \%)$ patients of Group B had shoulder stiffness, $2(8 \%)$ patients each of Group B had postoperative infection (5\%) and implant loosening. 2 (8\%) patients in Group A and Group B had malunion. There were no incidences of non-union or osteonecrosis in our study.

12. During the 6 months follow up period, $2(8 \%)$ patients of Group A and 1 (4\%) patient each of Group B had shoulder stiffness and implant loosening. 1 (4\%) patient in Group A and Group B had malunion. There was no significant association within the groups during 6 weeks and 6 months follow-up and also no significant association was observed between the groups as per ChiSquare test, $(p>0.05)$.

13. The range of motion at 6 Weeks and 6 months follow up is summarized in Table 12. The mean flexion, abduction, external rotation and internal rotation values of Group A were significantly lesser as compared to Group B after 6 weeks $(p<0.05)$. However there was no significant difference between the groups after 6 months. It was also observed that there was significant improvement in the range of motion within both the groups $(p<0.05)$.

14. During 6 weeks follow-up period, $2(8 \%)$ and $6(24 \%)$ patients in Group A and Group B respectively had excellent score while $10(40 \%)$ and $14(56 \%)$ patients had good score. Moderate score was observed in $8(32 \%)$ and $3(12 \%)$ patients respectively whereas poor score was observed in $5(20 \%)$ and $2(8 \%)$ patients.

15. During 6 months follow-up period, $7(28 \%)$ and $9(36 \%)$ patients in Group A and Group B respectively had excellent score while $14(56 \%)$ and $13(52 \%)$ patients had good score. Moderate score was observed in 3 (12\%) and $2(8 \%)$ patients respectively whereas poor score was observed in $1(4 \%)$ patient each. There was increase in the functional outcome of patients in both the groups but the increase was statistically not significant $(p>0.05)$.

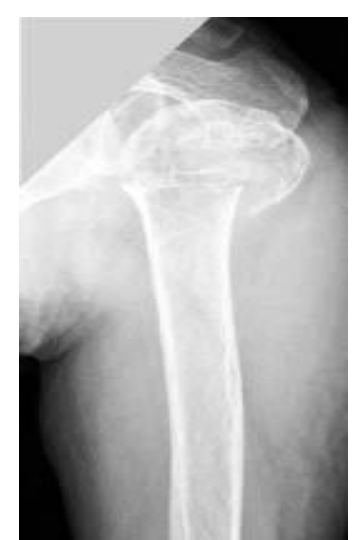

On Day of Trauma

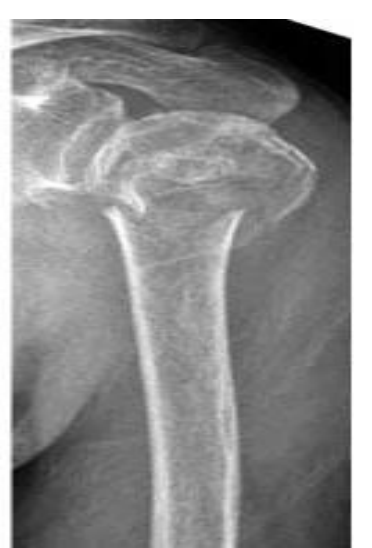

Post trauma 6 weeks

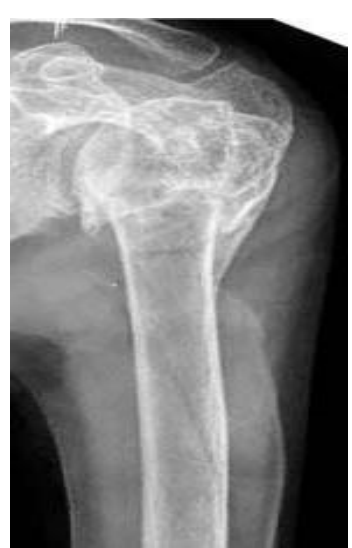

Post trauma 6 months 


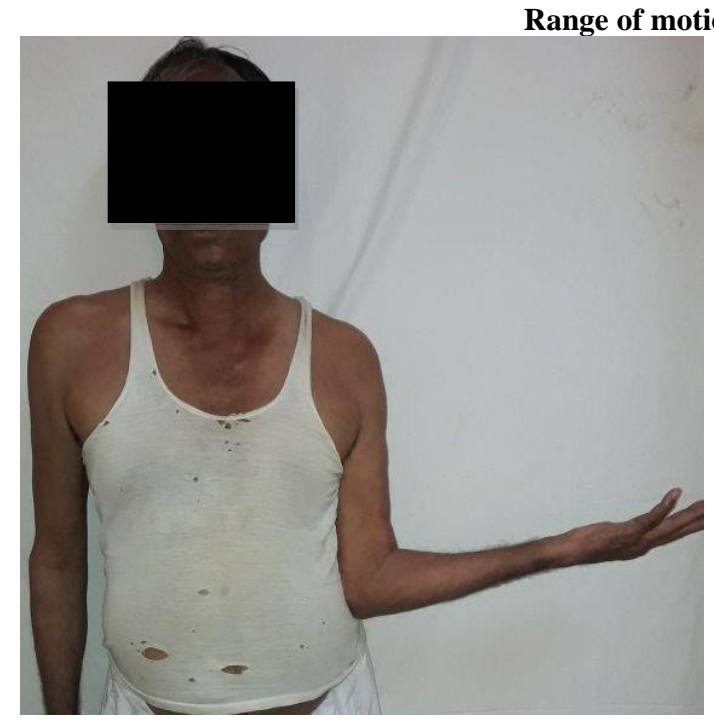

External Rotation

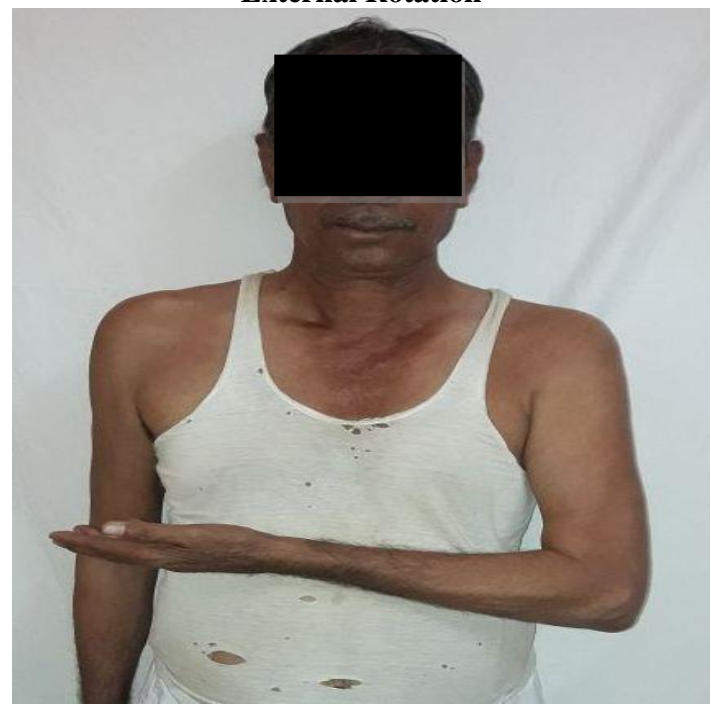

Internal Rotation
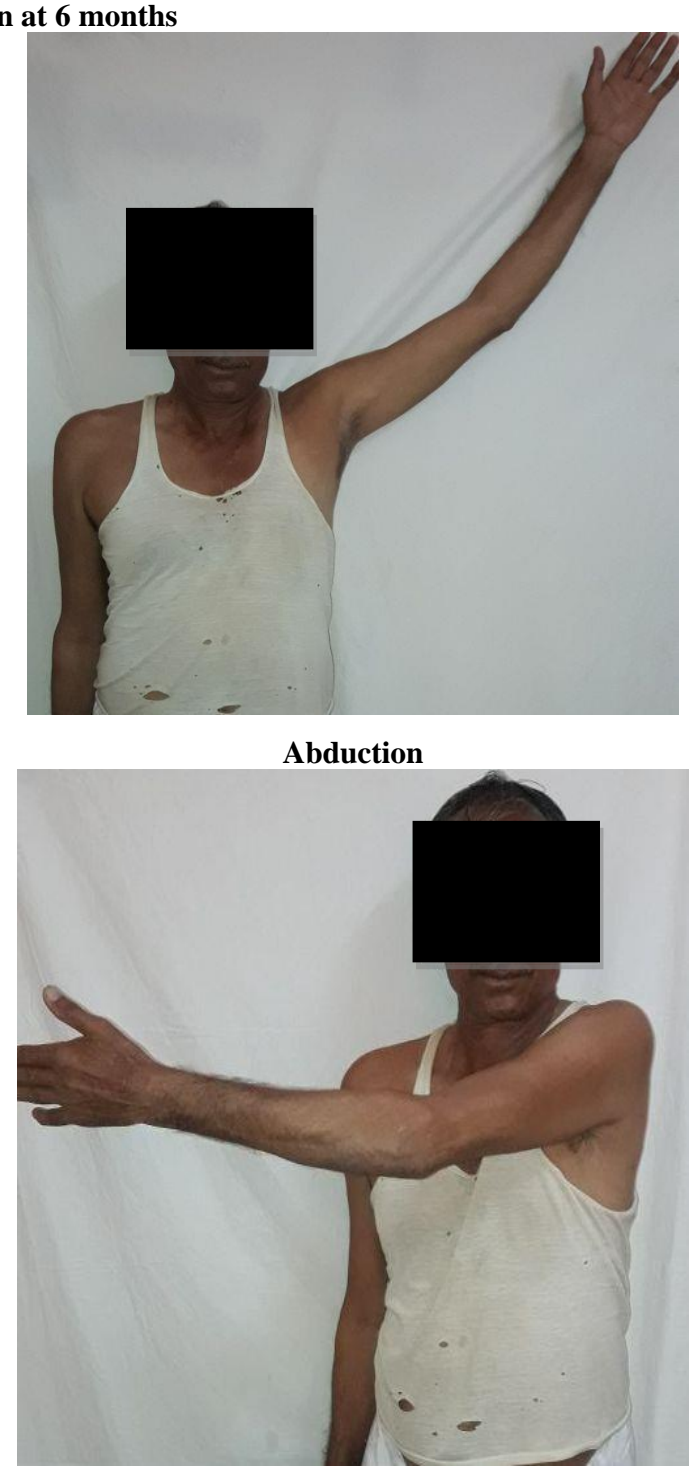

Flexion

Case 1: (Conservative Management)

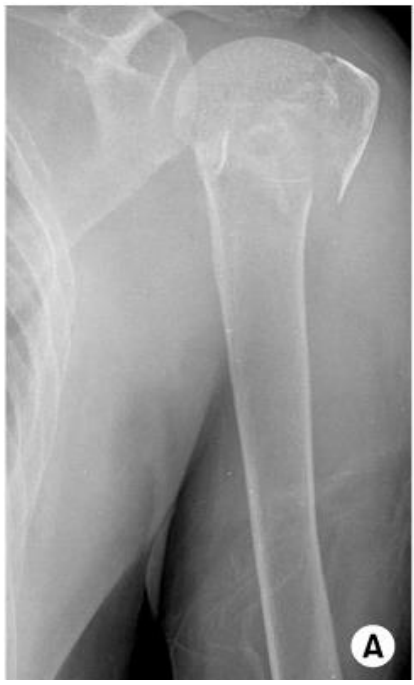

On Day of Trauma

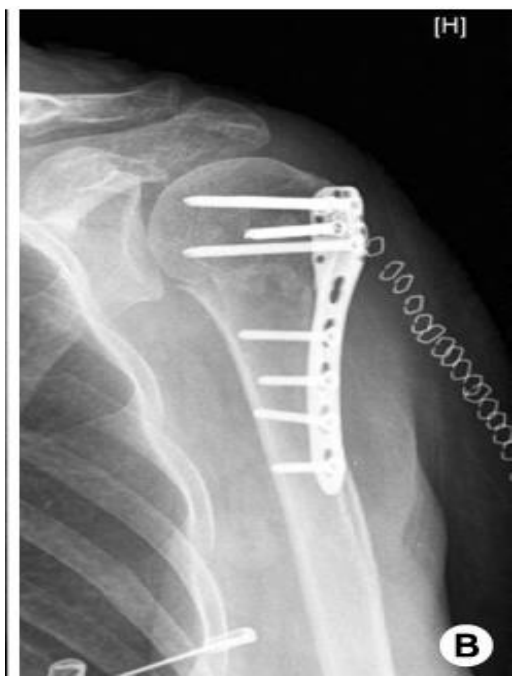

Post operative Day 1

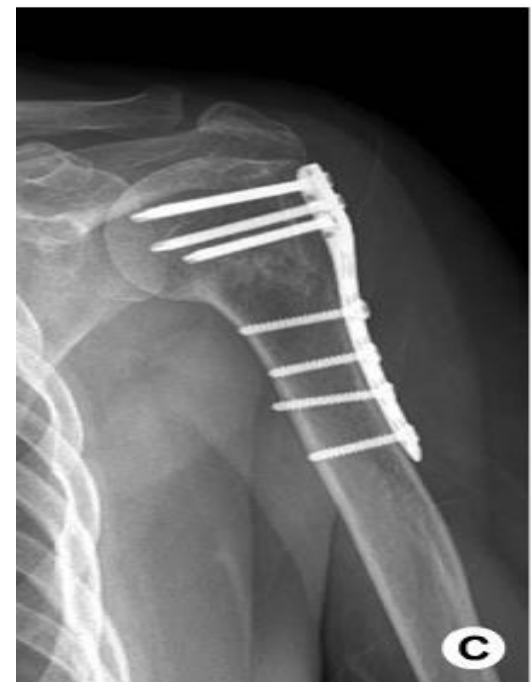

Post operative day 6 months 

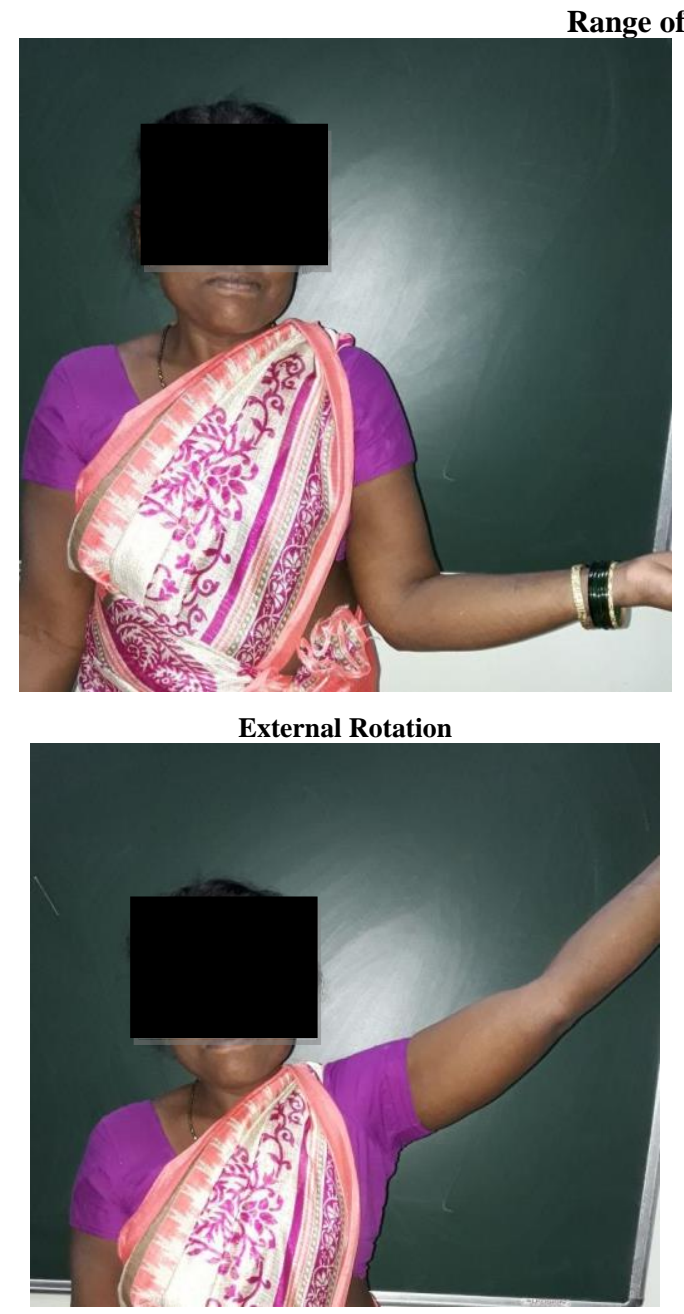

Abduction

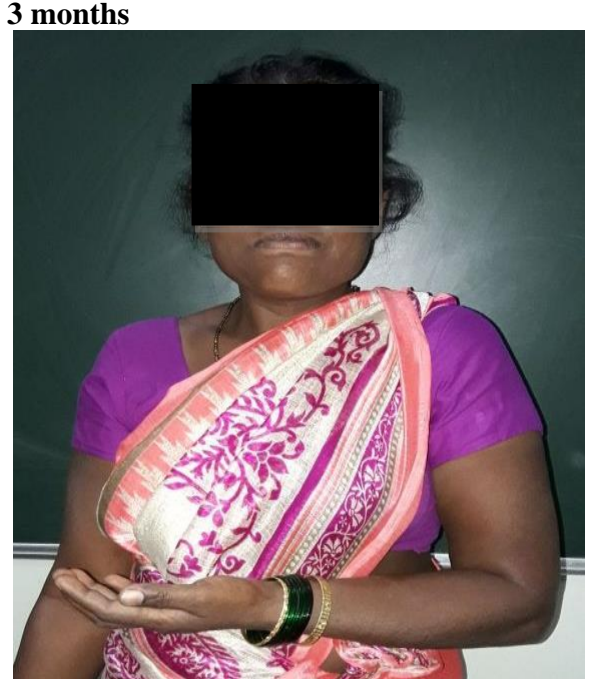

Internal Rotation

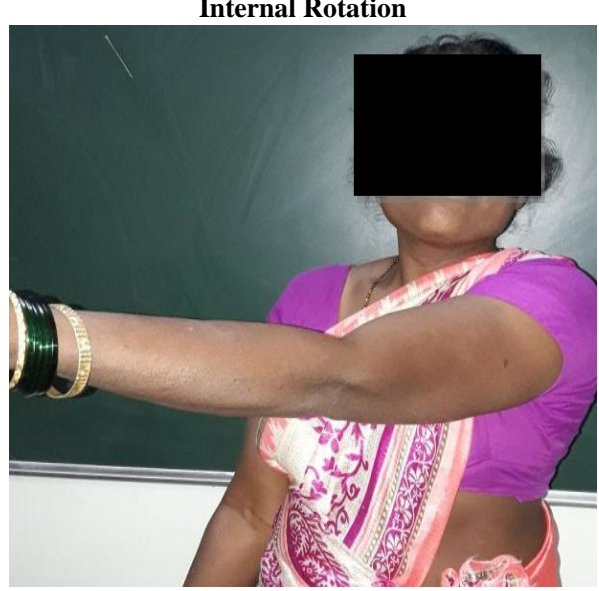

Flexion

Case 2: (Operative Management)

\section{Conclusion}

Locking plate is an implant of choice for comminuted proximal humerus fractures. Plates with attached (locked) screws may provide improved fracture stability and healing. Locking the screw to the plate mechanically recreates a point of cortical bone contact, which may be useful in the poor cancellous bone of the proximal humerus. Patients can allow early mobilization so less chances of joint stiffness. Due to proper reduction and alignment there is no chances of nonunion and malunion. But there is also some drawback of locking plate like it is costly and it is not available easily. In the present study, the outcomes of locked plate fixation were similar to those of nonoperative treatment. Early physiotherapy and good rehabilitation programme is vital to get a good functional outcome.

\section{References}

1. Horak J, Nilsson BE. Epidemiology of fracture of the upper end of the humerus. Clin Orthop Relat Res. 1975; 112:250-3.

2. Lind T, Kroner K, Jensen J. The epidemiology of fractures of the proximal humerus. Arch Orthop Trauma Surg. 1989; 108(5):285-7.

3. Crispin Ong. Christopher Bechtel. Three- and Four-part Fractures Have Poorer Function than One-part Proximal Humerus Fractures. Clin Orthop Relat Res. 2011; 469:3292-3299.

4. Barrett JA, Baron JA, Karagas MR, Beach ML. Fracture risk in the U.S. Medicare population. J Clin Epidemiol.
1999; 52:243-249.

5. Kristiansen B, Barfod G, Bredesen J, Erin-Madsen J, Grum B, Horsnaes MW et al. Epidemiology of proximal humeral fractures. Acta Orthop Scand. 1987; 58:75-7.

6. Jones G, Nguyen T, Sambrook P, Kelly PJ, Eisman JA. Progressive loss of bone in the femoral neck in elderly people: longitudinal findings from the Dubbo osteoporosis epidemiology study. BMJ. 1994; 309:691-5.

7. Bjorkenheim JM, Pajarinen J, Savolainen V. Internal fixation of proximal humeral fractures with a locking compression plate: a retrospective evaluation of 72 patients followed for a minimum of 1 year. Acta Orthop Scand. 2004; 75:741-745.

8. Gerber C, Werner CM, Vienne P. Internal fixation of complex fractures of the proximal humerus. J Bone Joint Surg Br. 2004; 86(6):848-855.

9. Strohm PC, Helwig P, Konrad G, Sudkamp NP. Locking plates in proximal humerus fractures. Acta Chir Orthop Traumatol Cech. 2007; 74:410-415.

10. Wijgman AJ, Roolker W, Patt TW, Raaymakers EL, Marti RK. Open reduction and internal fixation of three and four-part fractures of the proximal part of the humerus. J Bone Joint Surg Am. 2002; 84:1919-1925.

11. Antuna SA, Sperling JW, Cofield RH. Shoulder hemiarthroplasty for acute fractures of the proximal humerus: a minimum five-year follow-up. J Shoulder Elbow Surg. 2008; 17:202-209.

12. Dietrich M, Meier C, Lattmann T, Zingg U, Gruninger P, Platz A. [Complex fracture of the proximal humerus in 
the elderly: locking plate osteosynthesis vs hemiarthroplasty] [in German]. Chirurg. 2008; 79:231240.

13. Handschin AE, Cardell M, Contaldo C, Trentz O, Wanner GA. Functional results of angular-stable plate fixation in displaced proximal humeral fractures. Injury. 2008; 39:306-313.

14. Solberg BD, Moon CN, Franco DP, Paiement GD. Surgical treatment of three and four-part proximal humeral fractures. J Bone Joint Surg Am. 2009; 91:16891697.

15. Goldman RT, Koval KJ, Cuomo F, Gallagher MA, Zuckerman JD. Functional outcome after humeral head replacement for acute three- and four-part proximal humeral fractures. J Shoulder Elbow Surg. 1995; 4:81-86.

16. Agudelo J, Schurmann M, Stahel P, Helwig P, Morgan SJ, Zechel W et al. Analysis of efficacy and failure in proximal humerus fractures treated with locking plates. J Orthop Trauma. 2007; 21:676-681. 